\title{
For the Future of Clinics in Shoulder and Elbow
}

\author{
Sung-Jae Kim \\ Arthroscopy and Joint Research Institute, Department of Orthopaedic Surgery, Severance Hospital, Yonsei University College of Medicine, Seoul, Korea
}

Since its inauguration in 1993, the Korean Shoulder and Elbow Society (KSES) has showed remarkable growth for the last two decades. Although the history of shoulder and elbow surgery is relatively short in Korea, KSES has become a world-class society of shoulder and elbow surgery, comparable to those of Japan and other western countries. I believe that KSES is now one of the most promising orthopaedics societies and such development is all due to the active research and excellent presentation of our members.

KSES has actively held the annual meeting of shoulder and elbow surgery, related symposiums, and live surgeries and it certainly serves as an exemplary model to other societies in Korea. Especially, Clinics in Shoulder and Elbow (CiSE) has steadily developed into one of the leading orthopaedics journals in Korea; it is all due to active participation and research of our members.

Keeping pace with its recent development, the recent decision to switch CiSE into an English journal is timely and future-oriented for globalization of KSES and its further growth. I wish the transition can serve as a stepping point of being registered in the SCI journal list.

Furthermore, I believe that hosting the International Congress of Shoulder \& Elbow Surgery in 2016 in Jeju Island will raise the status of KSES to a higher level. I wish its great success and active participation of our members.

It is not much to say that my career as a (shoulder and elbow) surgeon has developed together with the growth of KSES. I wish further development of KSES in upcoming years, including being registered in the $\mathrm{SCl}$ journal list and holding many symposiums with world-class scholars.

Once again, I congratulate the transition of the Journal of the Korean Shoulder and Elbow Society to an English journal.

\author{
Correspondence to: Sung-Jae Kim \\ Arthroscopy and Joint Research Institute, Department of Orthopaedic Surgery, Severance Hospital, Yonsei University College of Medicine, 50-1 \\ Yonsei-ro, Seodaemun-gu, Seoul 120-749, Korea \\ Tel: +82-2-2228-5679, Fax: +82-2-363-6248, E-mail: sungjaekim@yuhs.ac
}

Financial support: None. Conflict of interests: None. 